\title{
Molecular cloning of a bovine immunoglobulin lambda chain cDNA
}

(Recombinant DN ; mammary gland; gene library; nucleotide sequence; pseudogene)

\author{
Vladimir N. Ivanov a, Vladimir A. Karginov'b, Igor V. Morozov ${ }^{\text {b }}$, Stanislav I. Gorodetsky ${ }^{\text {a }}$ \\ ${ }^{a}$ N.I. Vavilov Institute of General Genetics, USSR Academy of Sciences, Moscow (U.S.S.R.) and ${ }^{b}$ Institute of Bioorganic \\ Chemistry, USSR Academy of Sciences, Siberian Branch, 630090 Novosibirsk (U.S.S.R.) Tel. 35-6391
}

Received 1 December 1987

Accepted 25 December 1987

Received by publisher 21 March 1988

\section{SUMMARY}

A cDNA library of the bovine mammary gland constructed in pBR322 was screened by mRNA hybridselected translation and by differential hybridization. Several immunoglobulin (Ig) $\lambda$ light-chain clones were identified and sequenced. Nucleotide sequence comparison of bovine and human $\operatorname{Ig} \lambda$ chains showed a high degree of homology for constant regions and for $J$ regions. The amino acid (aa) sequence encoded by the constant region of the bovine Ig $\lambda$ chain cDNA contains 107 aa with differences at 24 aa positions from the human Ig $\lambda$ chain. Three complementarity-determining regions (CDR1, 2,3) characteristic of the variable region of bovine Ig $\lambda$ chain cDNA can be distinguished. The bovine and human sequences display good homology in the framework region 3 (FR3) but only patches of homology throughout the FR2 region. The $5^{\prime}$ end of the bovine Ig $\lambda$ chain cDNA fragment of clone 1-14E contains five stop codons: two in CDR1, one in FR1 and two in the hydrophobic prepeptide region. These data suggest that the $\operatorname{Ig} \lambda$ mRNA of clone $1-14 \mathrm{E}$ is transcribed from the $\mathrm{V}_{\lambda}$ pseudogene.

\section{INTRODUCTION}

Mammalian immunoglobulins are important components of colostrum and milk. The concentration of several classes of immunoglobulins in colostrum is higher than in serum. Species that transmit immunoglobulins exclusively in utero (man, rabbit) produce colostrum that is predominantly composed of $\operatorname{IgA}$, while those that transmit immunoglobulins exclu-

Correspondence to: Dr. V.N. Ivanov, N.I. Vavilov Institute of General Genetics, USSR Academy of Sciences, 3, Gubkin Street, 117809 Moscow (U.S.S.R.) Tel. 135-1229. sively via the colostrum (cattle, goat, pig), have colostrum predominantly composed of IgG. Bovine colostrum and milk also contain minor concentrations of SIgA and IgM. The immunoglobulins of the lacteal secretions are derived either from blood or from a local compartment of the immune system. The bulk of immunoglobulins is transferred into colostrum and milk transepithelially from the bloodstream. The local immune system of mammary

Abbreviations: aa, amino acid(s); bp, base pair(s); C, constant region; $\mathrm{CDR}$, complementarity-determining region; ds, double stranded; FR, framework region; Ig, immunoglobulin; nt, nucleotide(s); SDS, sodium dodecyl sulfate; sIgA, secretory immunoglobulin A; V, variable region. 
glands produces mainly sIgA. Antibodies of this class have a local protective anti-microbial function in mammary gland ducts and in the intestine of newborns (Butlcr, 1974; 1986).

At present, considerable information is available on organization and expression of immunoglobulin genes of mouse and man (Tonegawa, 1983; Honjo, 1983), although the information on bovine immunoglobulins is rather scarce. The amino acid sequence of bovine immunoglobulins and the nucleotide sequence of their mRNAs are unknown. We used a cDNA library of the bovine mammary gland constructed previously (Gorodetsky et al., 1985) for screening of $\operatorname{Ig} \lambda$ light-chain cDNA clones. As a result we isolated clones containing $\operatorname{Ig} \lambda$ chain $\mathrm{cDNA}$ fragments which were used for sequencing.

\section{MATERIALS AND METHODS}

\section{(a) Construction of cDNA library}

A cDNA library of the bovine mammary gland was constructed as described earlier (Gorodetsky et al., 1985). The ds cDNA and PstI-digested pBR322 were tailed with about $20 \mathrm{nt}$ of oligo(dC) and oligo(dG), respectively. After annealing, the recombinant plasmids were used to transform Escherichia coli JC5183 as described by Kushner (1978).

\section{(b) Isolation and analysis of recombinant DNA}

Plasmid DNA isolation, colony hybridization in situ, gel electrophoresis of DNA, nick translation, and blot hybridization were performed according to standard procedures (Maniatis et al., 1982). Hybridization selection-translation followed by $0.1 \%$ SDS- $12 \%$ polyacrylamide gel electrophoresis was performed as described previously (Ivanov et al., 1984).

\section{(c) Nucleotide sequencing}

Sequencing was performed by the method of Maxam and Gilbert (1980) after recloning the cDNA fragments into plasmids pUC19 or pGEM1.

\section{RESULTS}

(a) Analysis of the cDNA library of the bovine mammary gland

A minilibrary of 350 colonies carrying recombinant plasmids from the cDNA library of the bovine mammary gland were screened with ${ }^{32} \mathrm{P}$-labelled probes consisting of cloned fragments coding for $\alpha_{\mathrm{s} 1^{-}}, \beta$-, and $\kappa$-casein. Twelve, six and four colonies produced hybridization signals with these labelled probes, respectively. The rest of the minilibrary was collected into small groups including three or four clones. Plasmid DNA of these groups of clones was analyzed by mRNA hybrid-selected translation. This assay employed the plasmid DNA to select for specific mRNA by means of hybridization, followed by in vitro translation, immunoprecipitation, and $M_{\mathrm{r}}$ determination of the polypeptide. The immunoglobulin fraction of antiserum to total bovine milk proteins was used for immunoprecipitation. This antiserum contained antibodies to caseins, $\beta$-lactoglobulin and IgG. The translation products obtained from our clones are shown in Fig. 1. Lanes 2 and 3 correspond to proteins encoded by total unfractionated mRNA after immunoprecipitation with anti-milk protein antiserum. The mRNA which hybridized with plasmid DNA of one group of clones $(1-11 \mathrm{G}$, $1-13 \mathrm{U}$ and $1-15 \mathrm{~F}$ ) produced $\alpha_{\mathrm{s1}}$-casein, $\beta$-lactoglobulin and a $22-\mathrm{kDa}$ protein $\mathrm{p} 22$, whose electrophoretic mobility was in the region of $\beta$ - and $\kappa$-caseins (Fig. 1, lane 4). The next stage consisted of more accurate identification of individual clones. It was shown that the mRNA which hybridized with plasmid DNA of clones $1-15 \mathrm{~F}$ produced $\beta$-lactoglobulin, clone 1-11G, $\alpha_{\mathrm{s} 1}$-casein, and clone 1-13U, protein p22 (Fig. 1).

${ }^{32} \mathrm{P}$-labelled probes containing cloned fragments of $\beta$ - and $\kappa$-casein failed to hybridize with plasmid DNA of clone 1-13U. A preliminary assumption was that the cDNA fragment of clone 1-13U encoded immunoglobulin light chain because of its size of 22 $\mathrm{kDa}$ and electrophoretic mobility in the region of $\beta$ and $\kappa$-caseins. Since we did not have monospecific antisera to individual $\lambda$ and $\kappa$ light chains, identification of the fragment was performed by direct nucleotide sequencing. A $300-\mathrm{bp}$ PstI cDNA fragment of clone 1-13U was recloned into the Pstl site of plasmid pUC19 polylinker. 


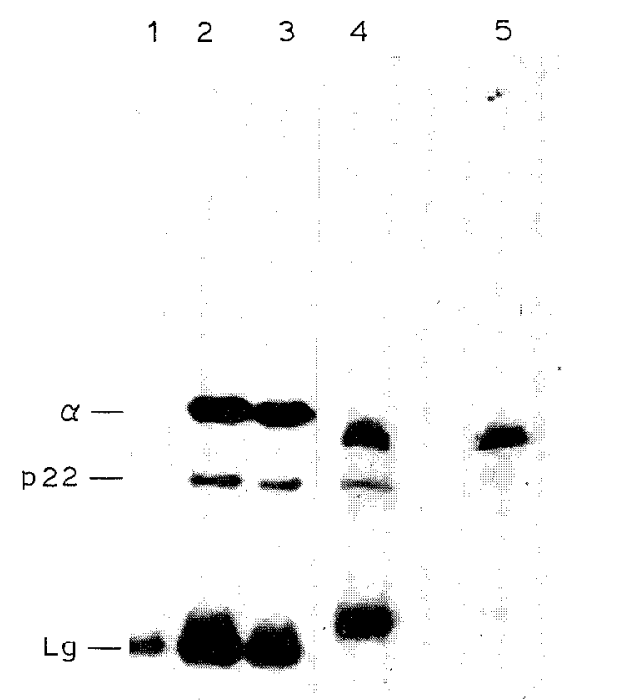

Fig. 1. Hybrid-selected translation. Translation products of total mammary gland mRNA following immunoprecipitation with antibodies to total milk proteins were used as a control (lanes 2 and 3). ${ }^{35}$ S-labelled translation products of mRNA selected by hybridization were immunoprecipitated and separated by electrophoresis in $0.1 \%$ SDS- $12 \%$ polyacrylamide gel. Total mRNA was hybridized with plasmid DNA of clone 1-15F (lane 1), a group of clones 1-13U, 1-15F and 1-11G (lane 4), and clone 1-11G (lane 5). Shown at the left are the main translation products of the mammary gland mRNA: $\alpha_{\mathrm{s} 1}$-casein $(\alpha)$, protein p22 (p22), $\beta$-lactoglobulin ( $\mathrm{Lg})$.

Sequencing has shown that this cDNA fragment encoded 67 aa with a high degree of homology to aa 150-217 of the human light chain and a portion of the 3 '-nontranslated region. The 300-bp Pst I cDNA fragment of clone 1-13U labelled by nick translation was used as a hybridization probe for screening of the minilibrary. About 30 colonies produced positive signals (Fig. 2). The size of cDNA fragments of these clones was usually from $200-400 \mathrm{bp}$. The largest was an 860-bp insert of clone 1-14E and a 560-bp insert of clone 1-130. The cDNA fragments of these clones were used for restriction map construction and sequencing.

To facilitate the sequencing, the fragments were recloned into the polylinker of plasmids pUC19 or pGEM1.

\section{(b) Sequencing of Ig $\lambda$ light-chain cDNA}

The restriction map of cDNA fragment of clone 1-14E and sequencing strategy are shown in Fig. 3. The exact size of this fragment was 862 bp with

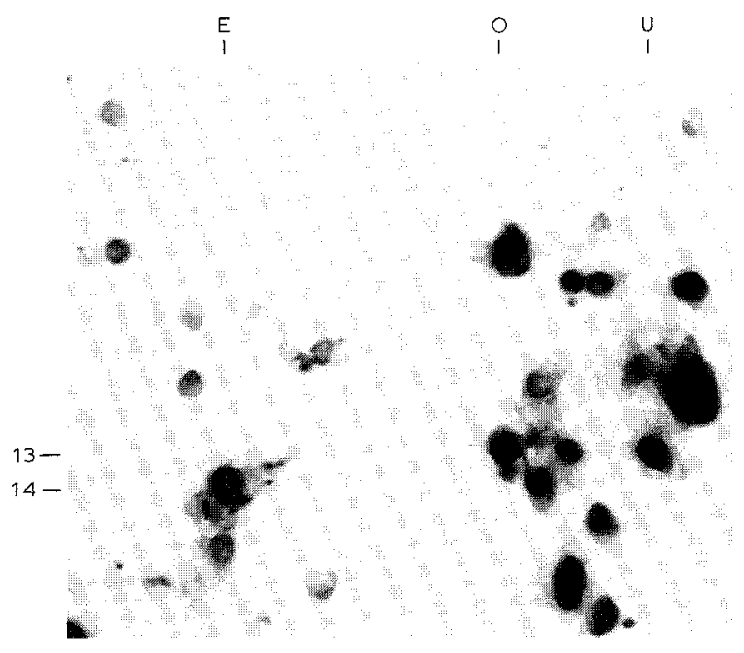

Fig. 2. Screening of the bovine mammary gland cDNA minilibrary. The 350 clones on nitrocellulose were numbered by columns designated $\mathrm{A}$ through $\mathrm{V}$ and rows designated 1 through 18 ; only columns $\mathrm{E}, \mathrm{O}, \mathrm{U}$ and rows 13 and 14 are marked on the margins. In situ colony hybridization was carried out with the ${ }^{32} \mathrm{P}$-labelled probe $\left(10^{6} \mathrm{cpm} / \mathrm{ml}\right)$, namely the $300-\mathrm{bp}$ Pst I fragment of clone 1-13U, which is the coding fragment of bovine $\operatorname{Ig} \lambda$ light-chain cDNA. Hybridization conditions were as described by Maniatis et al. (1982). All colonies of this minilibrary were given the prefix 1, e.g., 1-13U. Clones 1-13O, 1-13U and 1-14E were used for sequencing the bovine $\operatorname{Ig} \lambda$ chain $\operatorname{cDNA}$.

20-bp oligo(dG)-oligo(dC) connectors. Sequence comparison of human Ig $\lambda$ chain cDNA (Anderson et al., 1985) and a cDNA fragment of clone 1-14E revealed a considerable homology and allowed to identify this cloned fragment as bovine $\operatorname{Ig} \lambda$ chain cDNA (Figs. 4 and 5).

The complete $\mathrm{C}$ domain of bovine mature lightchain polypeptide is 107 aa long and is encoded by nt 420 through 740 . The constant regions of bovine and human cDNA differ only at 24 aa positions (Fig. 5). Therc is also a good homology between the $J$ region of human cDNA encoding aa 100-112 and the $\mathrm{J}$ region of bovine $\operatorname{Ig} \lambda$ cDNA (Fig. 4). Three complementarity-determining regions (CDR1, 2, 3) characteristic of V-region bovine $\mathrm{Ig} \lambda$ chain can be distinguished. The bovine and human sequences display good homology in the FR3 regions but only patches of homology throughout the FR2 regions. A poor homology is characteristic of the leader peptide regions and FR1 regions, although some aa residues (Ser-10, Gly-15, Gln-24) are conservative. We were surprised that the $5^{\prime}$-coding region of clone $1-14 \mathrm{E}$ cDNA contained five stop codons: two in the leaderpcptide rcgion, and one each in FR1 and in the 

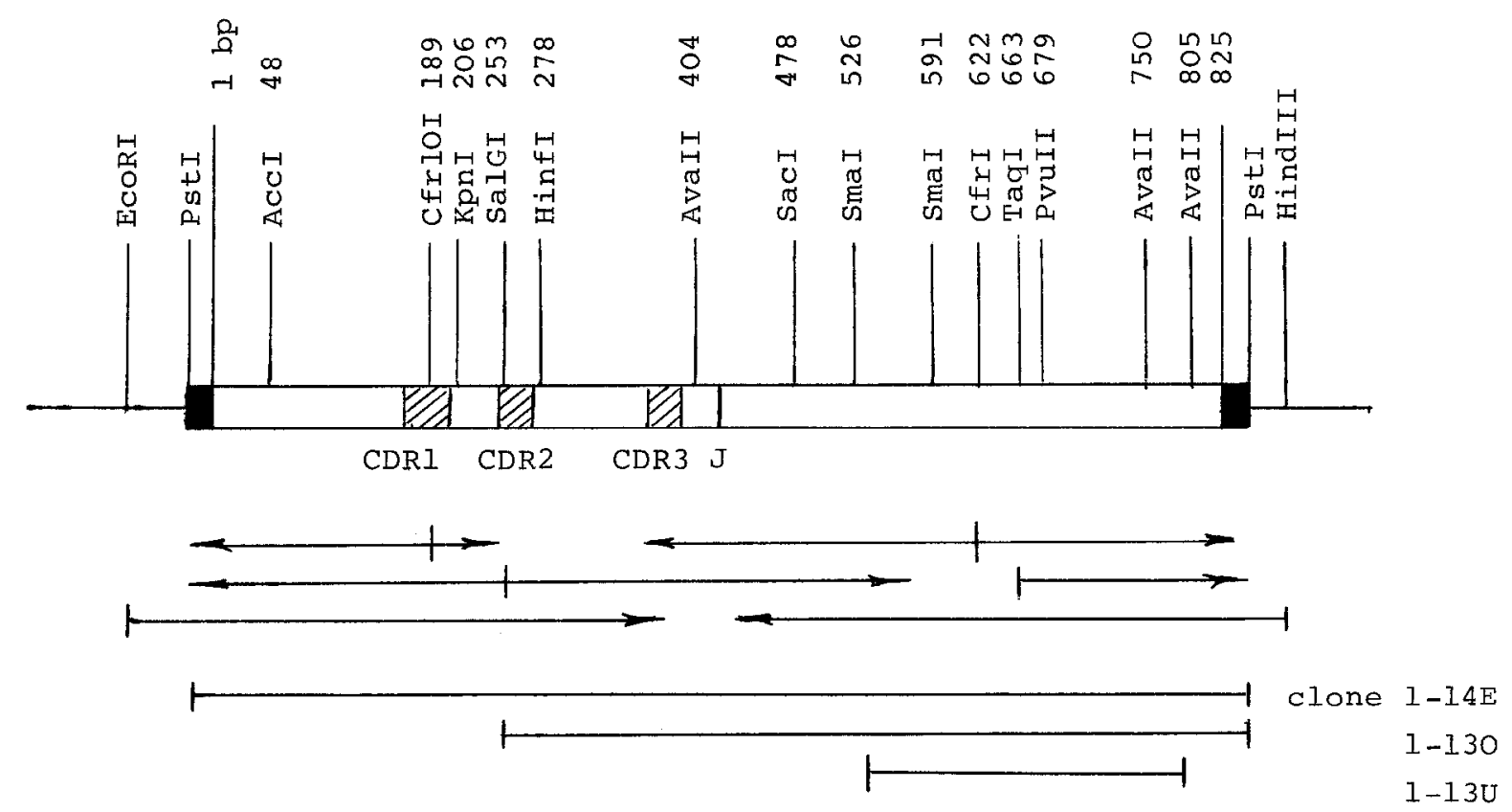

Fig. 3. Restriction map of Ig $\lambda$ chain cDNA (clone 1-14E) and sequencing strategy. The DNA restriction fragments indicated by arrows were sequenced by the procedure of Maxam and Gilbert (1980). The thin line represents the polylinker of pUC19; blackened boxes are oligo(dG)-oligo(dC) connectors, hatched boxes represent CDR segments, and open boxes are the other regions of Ig $\lambda$ light-chain $\mathrm{cDNA}$.

CDR1 regions. There was a small deletion in the leader peptide region and no Cys-22 at all. The existence of so many stop codons and other nucleotide sequence changes in the $5^{\prime}$-coding region of the cDNA fragment of clone 1-14E implied that the $\mathrm{V}$ region of this clone arose from a pseudogene. We performed additional screening of bovine mammary gland cDNA library containing about 5400 clones to find more Ig $\lambda$ cDNA clones with $5^{\prime}$ noncoding and $5^{\prime}$-coding regions. A ${ }^{32} \mathrm{P}$-labelled 270 -bp PstI-SalI subfragment of variable region of clone $1-14 \mathrm{E}$ was used as a probe. Twenty-four clones produced positive signals with this probe. The sizc of cDNA fragments was from 200 to $400 \mathrm{bp}$. Only three clones contained 860-bp cDNA fragments, namely clones $15 \mathrm{X}, 16 \mathrm{~F}$ and 23B. cDNA fragments of these clones were recloned into the PstI site of the polylinker of plasmid pGEM1 and used for sequencing. All the fragments had the same nucleotide sequence as the fragment of clone 1-14E with differences only in the connector size. The conclusion was that the nontranslated $\operatorname{Ig} \lambda$ mRNA which arose from a pseudogene was widespread in the mRNA population of the bovine mammary gland.

We also sequenced the 570-bp fragment of clone 1-130 encoding Ig $\lambda$ chain fragment starting at aa 53 .
Nucleotide sequence comparison of $\mathrm{V}$ regions of clones 1-130 and 1-14E is shown in Fig. 6. There are several differences in FR3, a lot of differences in the hypervariable region CDR3 and a few differences in the $J$ region. This suggests that cDNA of clones 1-130 and 1-14E arose from different $V_{\lambda}$ genes. Unfortunately, there is no information about the $5^{\prime}$ region of the second $\mathrm{V}_{\lambda}$ gene. At the same time, $\mathrm{C}$ regions of clones $1-14 \mathrm{E}$ and $1-130$ have the same sequence.

\section{DISCUSSION}

The complexity of the Ig $\lambda$ chain gene locus organization appears to correlate with the incidence of $\lambda$ chain polypeptides in immunoglobulin molecules. In inbred mice, immunoglobulins with $\kappa$ chains outnumber those with $\lambda$ chains by about $20: 1$. The $\lambda$ chain gene cluster is organized very simply and contains only $2 \mathrm{~V}_{\lambda}, 4 \mathrm{~J}_{\lambda}$ and $4 \mathrm{C}_{\lambda}$ genes (Tonegawa, 1983; Eisen and Reilly, 1985). About $40 \%$ of human immunoglobulins contain $\lambda$ light chain and the rest $\kappa$ light chain. Human Ig $\lambda$ chain gene locus organization is more complex. The number of $\mathrm{V}_{\lambda}$ genes is 
B 1

$\mathrm{H}$

B 45

B 90

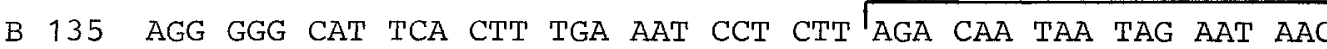
Arg Gly His Ser Leu xxx Asn Pro Leu Arg Gln xxx xxx Asn Asn 20

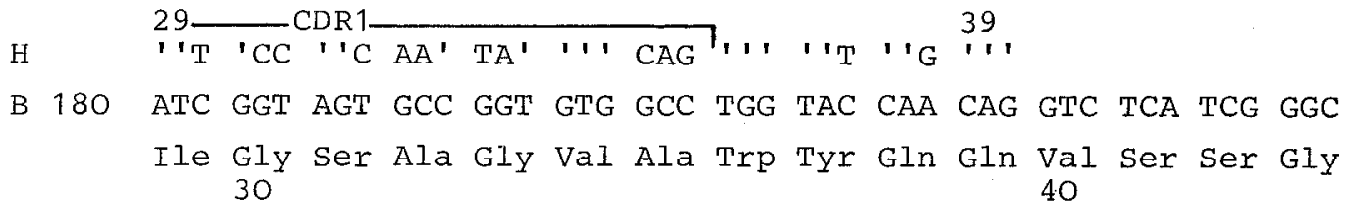

H $\quad 49, \ldots$, , '

B 225 CTC AGA ACC ATC ATC TAT GGT AGT AGT AGT CGA CCC TCG GGG GCT Leu Arg Thr Ile Ile Tyr Gly ser ser ser Arg Pro ser Gly Ala 50

H IT IT, וT, וT, $1,66 \quad 69$

B 270 CCA GAC CGA TTC TCC GGC TCA AAG TCT GGC ACT ACA GGC ACC CTG Pro Asp Arg Phe Ser Gly Ser Lys Ser Gly Thr Thr Ala Thr Leu
60

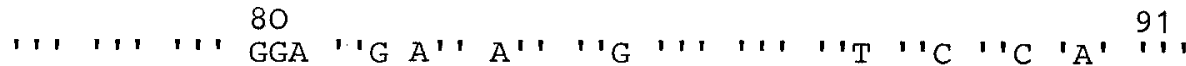

$\mathrm{H}$

B 315 ACC ATC ACC TCG CTC CAG GCT GAC GAC GAG GCG GAT TAT TTC TGT Thr Ile Thr Ser Leu Gln Ala Asp Asp Glu Ala Asp Tyr Phe Cys 80

II

B 360

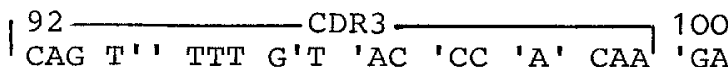

CAG T' TTT G'T 'AC 'CC 'A' CAA 'GA ' 'G ' 'C GCA ACT GCT GAC TAC AGT AGG AGT ACT GTT GTT TTT GGC AGC GGG Ala Thr Ala Asp Tyr ser Arg ser Thr val Val phe Gly ser Gly 90 ,' 'AG T', , ', 110

H

B 405 ACC AGA CTG ACC GTC Thr Arg Leu Thr VaI

Fig. 4. Comparison of the nucleotide sequences of the variable regions of the human (H) and bovine (B) Ig $\lambda$ chain cDNA (clone 1-14E). The human sequence is from Anderson et al. (1985). The prime marks indicate nucleotides common to both sequences. The nucleotides have been numbered for the bovine sequence starting at 1 (left margin). The corresponding amino acids are shown under the nucleotide sequence and are numbered starting at aa 1 ( $\mathrm{Val}$ ), which marks the beginning of the $\mathrm{V}$ region. Negative numbers refer to the amino acids of the leader sequence. The human nucleotide sequence is shown only for the region with high homology to the bovine sequence. Numbering of the codons is in accordance with the human Ig $\lambda$ amino acid scquencc (Anderson et al., 1985). The in-phase termination codons are marked xxx. 


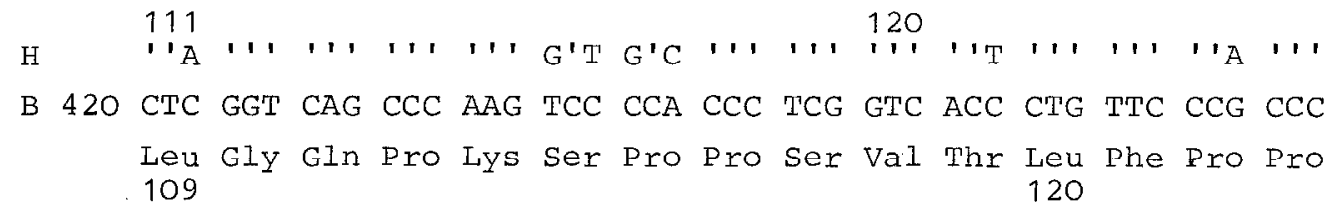

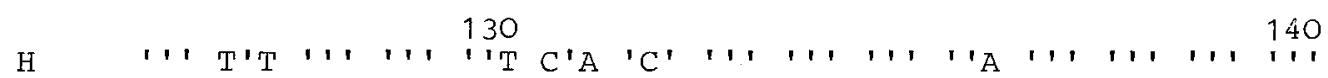

B 465 TCC ACG GAg GAg CTC AAC GGC AAC AAG GCC ACC CTG GTG TGT CTC Ser Thr Glu Glu Leu Asn Gly Asn Lys Ala Thr Leu Val Cys Leu 130

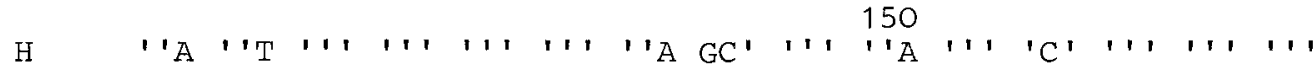

B 510 ATC AGC GAC TTC TAC CCG GGT AgC GTG ACC GTG GTC TGG AAg GCA Ile Ser Asp phe Tyr pro Gly ser val Thr Val Val Trp Lys Ala 140 150

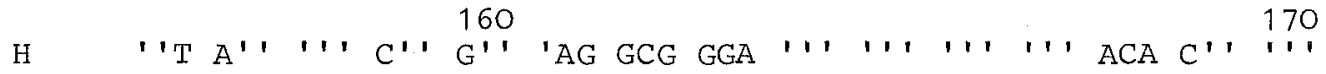

B 555 GAC GGC AGC ACC ATC ACC CGC AAC GTG GAG ACC ACC CGG GCC TCC Asp Gly Ser Thr Ile Thr Arg Asn Val Glu Thr Thr Arg Ala Ser 160

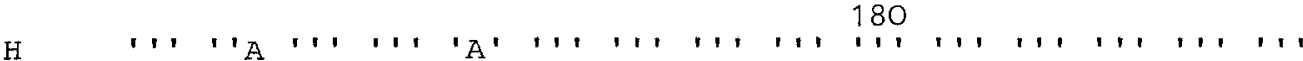

B 600 AAA CAG AGC AAC AGC AAg TAC GCC GCC AGC AGC TAC CTG AGC CTG Lys Gln Ser Asn Ser Lys Tyr ALa Ala Ser Ser Tyr Leu Ser Leu 170 180

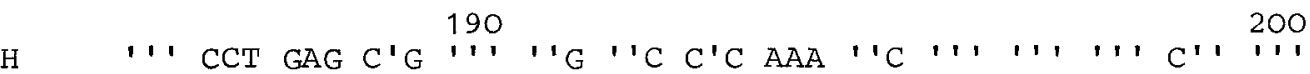

B 645 ACG AGC AGC GAC TGG AAA TCG AAA GGC AGT TAC AGC TGC GAG GTC Thr Ser Ser Asp Trp Lys Ser Lys Gly Ser Tyr Ser Cys Glu Val 190

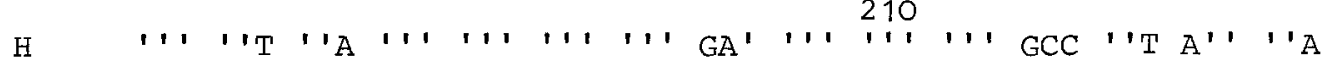

B 690 ACG CAC GAG GGG AGC ACC GTG ACG AAG ACA GTG AAG CCC TCA GAG Thr His Glu Gly Ser Thr Val Thr Lys Thr Val Lys pro ser Glu 200 210

217

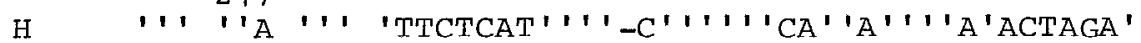

B 735 TGT TCT TAG GG---_-_CCCTGGACCCCCACCCTCGGGGGCCCTCTGGCCCACACC

Cys Ser Stop

215

\section{B 785 CCCTCCCCCACCTCTCCATGGACCCCTGAGCCCCTACCCAGGTCGCCTCACACAAGGGG \\ В 844 CCTCTCCCTCCCTCCCTGTTCCTGCTTCTC}

Fig. 5. Comparison of the nucleotide sequences of the constant region of the human $(\mathrm{H})$ and bovine (B) Ig $\lambda$ chain cDNA (clone 1-14E). The human sequence is from Anderson et al. (1985). The prime marks indicate nucleotides common to both sequences. Dashes represent the gaps introduced to maximise homologies. The nucleotides have been numbered for bovine sequence starting at 420 (left margin). The corresponding amino acids are shown under the nucleotide sequence and are numbered starting at aa 109 (Leu). The human Ig $\lambda$ nucleotide sequence codons are shown above the bovine sequence and are numbered according to the human Ig $\lambda$ amino acid sequence. The last $47 \mathrm{nt}$ of the $3^{\prime}$-noncoding region of bovine $\operatorname{Ig} \lambda \mathrm{cDNA}$ are from clone 1-130. 


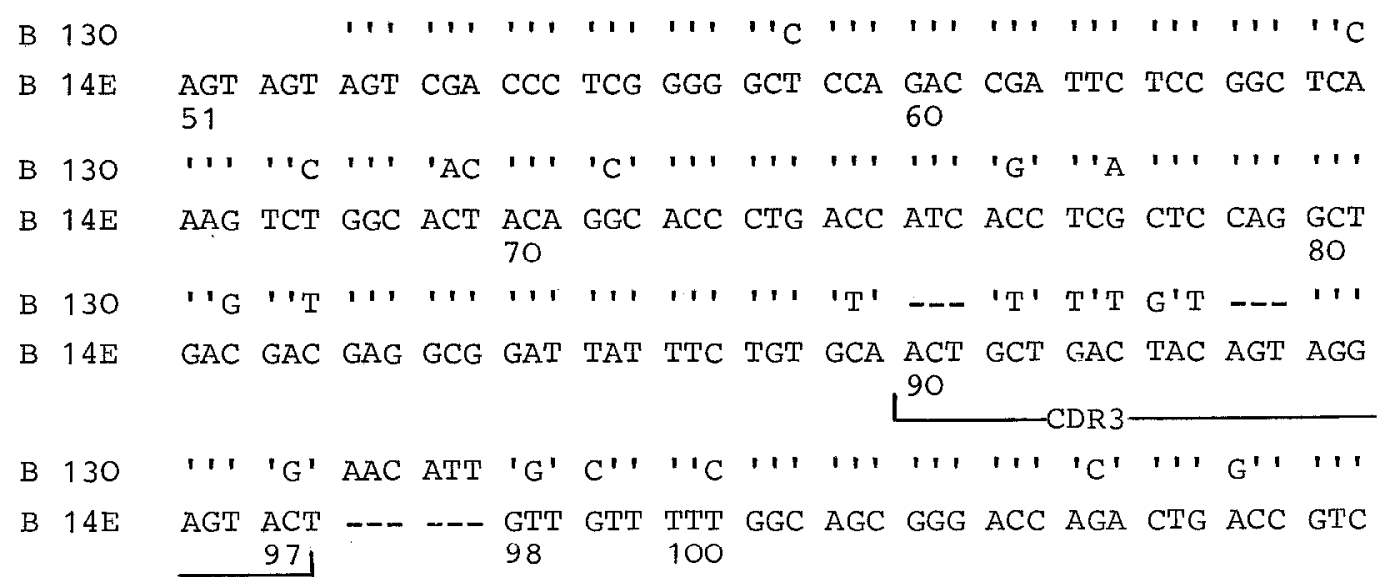

Fig. 6. A comparison of the nucleotide sequence of variable regions of bovine $\operatorname{Ig} \lambda$ chain cDNA fragments from clones 1-130 and 1-14E. The prime marks indicate nucleotides common to both sequences; dashes represent the gaps introduced to maximise homologies. The bovine nucleotide sequence codons (clone 1-14E) are numbered in accordance with Fig. 4.

15 to 20 (Anderson et al., 1985). Finally, more than $90 \%$ of bovine immunoglobulins contain $\lambda$ light chains (Butler, 1986). The bovine $\lambda$ chain locus has probably the most complex organization.

The Ig gene family is rich in pseudogenes. The $\mathrm{V}_{\mathrm{H}}$ locus of the human genome contains about $40 \%$ pseudogenes and one of its clusters up to $70 \%$ pseudogenes (Kodaira et al., 1986). Numerous pseudogenes are characteristic of the chicken $\lambda$ chain gcne family containing $15 \mathrm{~V}_{\lambda}$ gene (Reynaud et al., 1985; Parvari et al., 1986). Maybe the bovine $\lambda$ gene family is also rich in pseudogenes. The evidence about pseudogene origin of the mRNA of clone $1-14 \mathrm{E}$ is not surprising, but it is very interesting that this messenger is widespread in the total mRNA population of bovine mammary gland. $A$ different hypothesis may be proposed to explain these data, including the loss of the pseudogene's ability to regulate transcription, and a change in stability of the mRNA transcribed from the pseudogene. The one that seemed interesting to us is the reading of pseudogene mRNA in a wrong frame. Indeed, in case of frameshift it is possible to translate polypeptide $X$ containing 28 aa starting from the ATG codon (nt $77-79$ ) to the stop codon (nt 160-162). This polypeptide would be rich in serine and have some degree of homology with the polyserine cluster of bovine $\beta$-casein (Fig. 7). One of the main functions of $\beta$ -

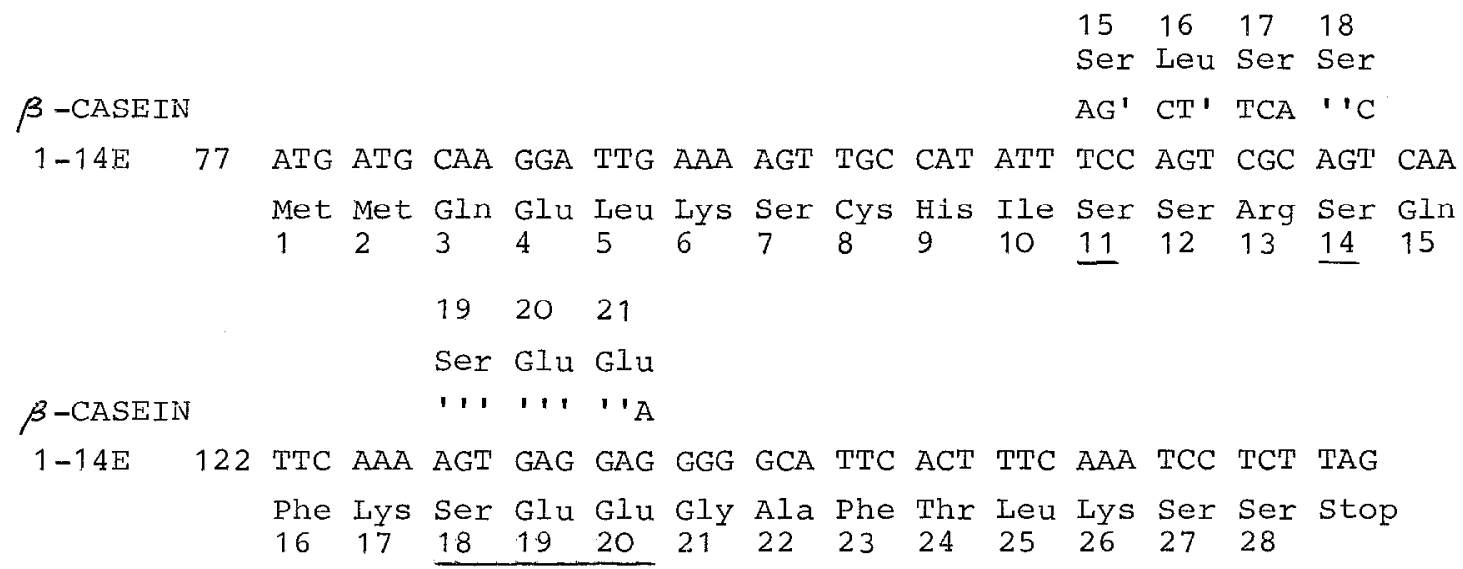

Fig. 7. Bovine Ig $\lambda$ chain mRNA (clone 1-14E) read in a wrong frame starting from $\mathrm{nt} 77$. The deduced amino acid sequence of polypeptide $\mathrm{X}$ is shown under the nucleotide sequence. The homology region with the polyserine cluster of bovine $\beta$-casein (Bayev et al., 1987) is underlined. 
casein is to transfer phosphate and calcium by serine-residue phosphorylation. Hypothetic polypeptide $X$ would thus serve as an additional phosphate and calcium transfer agent for the developing organism. If this hypothesis were proved by direct evidence on the existence of this polypeptide in the bovine mammary gland it would be an indication of the molecular substitution of some immunoglobulin pseudogenes as a result of a high mutation hypervariability of these genes.

\section{ACKNOWLEDGEMENTS}

We are grateful to N.P. Mertvetsov for helpful discussions.

\section{REFERENCES}

Anderson, M.L.M., Brown, L., McKenzie, E., Kellow, J.E. and Joung, B.D.: Cloning and sequence analysis of an $\operatorname{Ig} \lambda$ light chain mRNA in the Burkitt's lymphoma cell line EB4. Nucl. Acids Res. 13 (1985) 2931-2941.

Bayev, A.A., Smirnov, I.K. and Gorodetsky, S.I.: The primary structure of bovine $\beta$-casein cDNA. Mol. Biol. (in Russian) 20 (1987) 255-265.

Butler, J.E.: Biochemistry and biology of ruminant immunoglobulins. In Pandey, R. (Ed.), Progress in Veterinary Microbiology and Immunology, Vol. 2. Karger, Basel, 1986, pp. $1-52$.

Butler, J.E.: Immunoglobulins of the mammary secretions. In Larson, B.L. and Smith, V.R. (Eds.), Lactation, Vol. 3. Academic Press, New York, 1974, pp. 217-255.
Eisen, H.N. and Reilly, E.B.: Lambda chains and genes in inbred mice. Annu. Rev. Immunol. 3 (1985) 337-365.

Gorodetsky, S.I., Kershulite, D.R., Ivanov, V.N., Kapelinskaya, T.V. and Kaledin, A.S.: Synthesis and cloning of DNA complementary to mRNA from bovine mammary gland. Genetika 21 (1985) 191-200.

Honjo, T.: Immunoglobulin genes. Annu. Rev. Immunol. 1 (1983) $499-528$.

Ivanov, V.N., Kershulite, D.R., Bayev, A.A., Akhundova, A.A. Sulimova, G.E., Judinkova, E.S. and Gorodetsky, S.I.: Identification of bacterial clones encoding bovine caseins by direct immunological screening of the cDNA library. Gene 32 (1984) 381-388.

Kadaira, M., Kinashi, T., Umemura, I., Matsuda, F., Noma, T., Ono, Y. and Honjo, T.: Organization and evolution of variable region genes of the human immunoglobulin heavy chain. J. Mol. Biol. 190 (1986) 529-541.

Kushner, S.R.: An improved method for transformation of $E s$ cherichia coli ColE1-derived plasmids. In Boyer, H.W. and Nicosia, S. (Eds.), Genetic Engineering. Elsevier, Amsterdam, 1978, pp. 17-23.

Maniatis, T., Fritsch, E.F. and Sambrook, J.: Molecular Cloning. A Laboratory Manual. Cold Spring Harbor Laboratory, Cold Spring Harbor, NY, 1982.

Maxam, A.M. and Gilbert, W.: Sequencing end-labelled DNA with base-specific chemical cleavages. Methods Enzymol. 65 (1980) 499-560.

Parvari, R., Zim, E., Lentner, F., Ter-Or, S., Burstein, Y. and Schechter, I.: Analyses of chicken immunoglobulin light chain cDNA clones indicate a few germline $\mathrm{V}_{\lambda}$ genes and allotypes of $\mathrm{C}_{\lambda}$ locus. EMBO J. 6 (1987) 97-102.

Reynaud, C.-A., Anquez, V., Dahan, A. and Well, J.-C.: A single rearrangement event generates most of the chicken immunoglobulin light chain diversity. Cell 40 (1985) 283-291.

Tonegawa, S.: Somatic generation of antibody diversity. Nature 302 (1983) 575-581.

Communicated by A.A. Bayev. 